\title{
Language-level Symmetry Reduction for Probabilistic Model Checking
}

\author{
Alastair F. Donaldson \\ Computing Laboratory \\ Oxford University \\ Oxford, UK \\ Email: alastair.donaldson@comlab.ox.ac.uk
}

\author{
Alice Miller \\ Department of Computing Science \\ University of Glasgow \\ Glasgow, UK \\ Email:alice@dcs.gla.ac.uk
}

\author{
David Parker \\ Computing Laboratory \\ Oxford University \\ Oxford, UK \\ Email:david.parker@comlab.ox.ac.uk
}

\begin{abstract}
Symmetry reduction is a technique for combating state-space explosion in model checking. The generic representatives approach to symmetry reduction uses a language-level translation of symmetric models to a reduced form, making it straightforward to combine with existing tools and implementations. These techniques have been proposed for both non-probabilistic and probabilistic model checking, but are currently difficult to apply to complex models due to prohibitive restrictions in the modelling language. We present a much richer language, which allows specification of probabilistic systems in a way that guarantees the applicability of the generic representatives technique, together with an extended translation algorithm, and demonstrate the effectiveness of our techniques on a large set of case studies.
\end{abstract}

Keywords-Symmetry reduction; probabilistic model checking; counter abstraction

\section{INTRODUCTION}

Probabilistic model checking provides a powerful set of techniques for formally verifying quantitative properties of systems that exhibit stochastic behaviour. As with any exhaustive approach to formal verification, when applying these methods to real-life systems it quickly becomes essential to use techniques that improve the efficiency of the process. For probabilistic model checking, the need for such techniques is particularly acute since it requires not only an exhaustive state-space exploration, but also a numerical solution phase to compute probabilities or other quantitative values. Possible approaches include generating a compact model representation, e.g. with state compaction or symbolic (BDD-based) data structures, and reducing the size of the model to be analysed, e.g. via abstraction, bisimulation minimisation, partial order or symmetry reduction.

In this paper, we consider symmetry reduction [1], [2], [3], [4], which exploits the presence of replication in a system. Symmetry is in fact prevalent in many of the application domains to which probabilistic model checking has already proved valuable, e.g. randomized distributed algorithms, communication protocols and biological systems.

Symmetry reduction via generic representatives [5], [6] combines the benefits of symmetry reduction and symbolic representation. A system specification is translated into a reduced form with a set of counters that represent the behaviour of processes generically, keeping track how many processes reside in each local state, but not of individual process identities. The semantics of the generic specification is isomorphic to the symmetry-reduced model associated with the original specification. In most cases, the new model is not only significantly smaller, but also has a more compact symbolic representation. This is particularly beneficial in the context of probabilistic model checking since, for many state-of-the-art implementations (including the PRISM tool, to which we apply our techniques), both model size (number of states) and the size of a symbolic representation are critical. Initial results applying symmetry reduction by generic representatives to probabilistic model checking indicate that this is indeed the case [7], [8].

Although effective for certain examples, the original generic representatives technique has limited application: it can only be applied to a single family of identical processes (or one with an additional singleton process [6]) where the local state of each process is modelled by a single variable.

In this paper we significantly extend the application of generic representatives. We expand the theory to specifications consisting of multiple families of identical processes, whose state can be represented by multiple local variables, and whose behaviour can be described by guarded commands involving the kind of complex expressions over local and global variables required to model realistic systems.

We present our results by introducing Symmetric Probabilistic Specification Language (SPSL), a modelling language for symmetric systems with shared-variable communication, designed to guarantee applicability of the generic representatives approach. We focus on a Markov decision process (MDP) semantics for SPSL. Since MDPs generalise Kripke structures, SPSL also represents a significant improvement to the applicability of generic representatives to non-probabilistic model checking.

We present an algorithm for translating SPSL specifications to generic form, such that the symmetry-reduced semantics can be analysed using the PRISM model checker [9]. We have implemented the translation in a tool, GRIP, and have applied it to a wide range of case studies. We provide a comprehensive experimental evaluation, illustrating that our techniques provide excellent improvements in efficiency, both in terms of the feasibility of verifying large 
models and the time required for model construction and analysis. We also study the relative merits of a complementary symmetry reduction approach for PRISM [10].

\section{SYMMETRY REDUCTION FOR MDPS}

We review some background material on Markov decision processes (MDPs), symmetry reduction and, in particular, generic representatives.

\section{A. Markov decision processes}

We use $\operatorname{Dist}(S)$ to denote the set of discrete probability distributions over a set $S$.

Definition 1: A Markov decision process (MDP) is a tuple $\mathcal{M}=\left(S, s_{0}\right.$, Steps $)$, where $S$ is a finite set of states, $s_{0} \in S$ an initial state, and Steps $: S \rightarrow 2^{\operatorname{Dist}(S)}$ a probabilistic transition function.

An MDP $\mathcal{M}$ represents a system which exhibits both nondeterministic and probabilistic behaviour (for example, the asynchronous parallel composition of a set of randomized processes). Intuitively, in each state $s \in S$, there is a nondeterministic choice between the elements of Steps $(s)$. The next state of the model to which a transition will occur is then chosen probabilistically according to the selected distribution $\mu \in \operatorname{Steps}(s)$. A path through an MDP is obtained by resolving both probabilistic and non-deterministic choices. To do this one assumes that non-deterministic choices are resolved by an adversary which selects a choice based on the history of choices so far. A probability measure is defined that allows one to calculate the maximal and minimal probability for a set of paths.

\section{B. Automorphisms and quotient MDPs}

Definition 2: Let $\mathcal{M}=\left(S, s_{0}\right.$, Steps $)$ and $\mathcal{M}^{\prime}=$ $\left(S^{\prime}, s_{0}^{\prime}\right.$, Steps $\left.^{\prime}\right)$ be MDPs, and $\alpha: S \rightarrow S^{\prime}$ a bijection. Suppose $\alpha\left(s_{0}\right)=s_{0}^{\prime}$, and for $s \in S, \mu \in \operatorname{Steps}(s)$ iff there exists $\mu^{\prime} \in$ Steps $^{\prime}(\alpha(s))$ such that, for all $t \in S$, $\mu(t)=\mu^{\prime}(\alpha(t))$. Then $\alpha$ is an isomorphism from $\mathcal{M}$ to $\mathcal{M}^{\prime}$, and $\mathcal{M}$ and $\mathcal{M}^{\prime}$ are said to be isomorphic.

Definition 3: An automorphism of $\mathcal{M}$ is an isomorphism from $\mathcal{M}$ to $\mathcal{M}$. The set of all automorphisms of $\mathcal{M}$ forms a group under composition of mappings, denoted $\operatorname{Aut}(\mathcal{M})$.

Definition 4: Let $G \leq A u t(\mathcal{M})$. The orbit relation for $G$ is the set $\theta=\{(s, \alpha(s)): s \in S, \alpha \in G\} \subseteq S \times S$. For $s \in$ $S$, the orbit of $s$ under $G$ is the set $[s]_{G}=\{t:(s, t) \in \theta\}$. If $G$ is clear from the context, we write $[s]$ rather than $[s]_{G}$.

Definition 5: Suppose that we have a total ordering for $S$ and let $\min [s]$ denote the smallest element of $[s]$ for any state $s$. For any $G \leq A u t(\mathcal{M})$, the quotient $M D P$ for $\mathcal{M}$ w.r.t. $G$ is the MDP $\overline{\mathcal{M}}=\left(\bar{S}, \overline{s_{0}}, \overline{\text { Steps }}\right)$ where:

- $\bar{S}=\{\min [s]: s \in S\}$ and $\overline{s_{0}}=\min \left[s_{0}\right]=s_{0}$
- for each $\min [s] \in \bar{S}$ and $\mu \in \operatorname{Steps}(\min [s])$, $\overline{\text { Steps }}(\min [s])$ contains a distribution $\bar{\mu} \in \operatorname{Dist}(\bar{S})$ where, for $\min [t] \in \bar{S}, \bar{\mu}(\min [t])=\sum_{x \in[t]} \mu(x)$.

We follow the widely used convention of choosing $\min [s]$ as a unique representative of $[s]$.

Let $A$ be a set which we call atoms. In our application domain, an atom is a boolean expression over variables of a concurrent system. The logic PCTL (probabilistic computation tree logic) [11], [12] is defined as a set of state and path formulae over a set of atoms $A$. If $s$ is a state of MDP $\mathcal{M}$ and $a \in A$ is an atom, then $\mathcal{M}, s=a$ if $a$ evaluates to true at $s$. If $\bowtie \in\{<, \leq,>, \geq\}, p \in[0,1]$ and $\psi$ is a path formula, then $\mathcal{M}, s \models P_{\bowtie p}[\psi]$ if the probability $q$ of a path from $s$ satisfying $\psi$ is such that $q \bowtie p$ for all adversaries. If $s_{0}$ is the initial state of $\mathcal{M}$ and $\phi$ is a PCTL formula, we write $\mathcal{M}=\phi$ to denote that $\mathcal{M}, s_{0} \models \phi$. For full details on PCTL, see [11], [12].

The following theorem, proved in [13], establishes a correspondence between PCTL properties of isomorphic MDPs under an appropriate transformation of atoms.

Theorem 1: Let $\mathcal{M}=\left(S, s_{0}\right.$, Steps $)$ and $\mathcal{M}^{\prime}=$ $\left(S^{\prime}, s_{0}^{\prime}\right.$, Steps $\left.{ }^{\prime}\right)$ be MDPs, $A$ and $A^{\prime}$ sets of atoms, $\gamma: A \rightarrow$ $A^{\prime}$ a bijection, and $\delta$ an isomorphism from $\mathcal{M}$ to $\mathcal{M}^{\prime}$ such that, for every $s \in S$ and $a \in A, \mathcal{M}, s \models a \Leftrightarrow \mathcal{M}^{\prime}, \delta(s) \models$ $\gamma(a)$. Then for any PCTL formula $\phi$ over $A$ and $s \in S$,

$$
\mathcal{M}, s \models \phi \Leftrightarrow \mathcal{M}^{\prime}, \delta(s) \models \gamma(\phi)
$$

where $\gamma(\phi)$ is the PCTL formula over $A^{\prime}$ obtained by replacing every atom $a$ occurring in $\phi$ with $\gamma(a)$.

For $G \leq \operatorname{Aut}(\mathcal{M})$ and $\phi$ a $P C T L$ formula, we say that $\phi$ is symmetric with respect to $G$ if, for every maximal propositional sub-formula $f$ appearing in $\phi$ and for any state $s \in S$, we have $\mathcal{M}, s=f \Rightarrow \bigwedge_{s^{\prime} \in[s]} \mathcal{M}, s^{\prime} \mid=f$ [2].

The following theorem, proved in [13], shows that if $\phi$ is a PCTL formula which is symmetric with respect to a group of MDP automorphisms then we can check whether $\phi$ holds for $\mathcal{M}$ by considering the quotient MDP $\overline{\mathcal{M}}$ (which may be considerably smaller than $\mathcal{M}$ ).

Theorem 2: Let $\phi$ be a PCTL formula which is symmetric w.r.t. $G \leq A u t(\mathcal{M})$. Let $\overline{\mathcal{M}}$ be the quotient MDP for $\mathcal{M}$ w.r.t. $G$. Then $\mathcal{M}=\phi \Leftrightarrow \overline{\mathcal{M}}=\phi$.

For any $s \in S$, the size of $[s]$ is bounded by $|G|$, so the theoretical minimum size of $\bar{S}$ is $|S| /|G|$. For highly symmetric systems with $n$ components we may have $|G|=$ $n$ !, in which case exploiting symmetry potentially offers a considerable reduction in memory requirements.

\section{Generic representatives}

In the context of symbolic model checking, construction and representation of the orbit relation can be very costly. The generic representatives method of symmetry reduction 
avoids construction of the orbit relation by applying symmetry at the language level via a source-to-source translation [5], [6], and thus requires no modification of existing model checking algorithms.

The idea is best explained using an example. Consider a mutual exclusion algorithm for three identical processes, each with 3 local states neutral $(N)$, trying $(T)$ and critical $(C)$. The global states $(N, N, T),(T, N, N)$ and $(N, T, N)$ are symmetrically equivalent and have generic representative $(2 N, 1 T)$. A generic representative indicates how many processes are in each local state, without referring to individual processes.

The technique involves translating a fully symmetric specification $\mathcal{P}$, consisting of multiple instances of a single process type, into a generic form $\mathfrak{h}(\mathcal{P})$ where processes are replaced by counters. In $\mathfrak{h}(\mathcal{P})$ a counter variable is defined for each process local state indicating the number of processes currently in that state. Translation rules ensure that the model $\mathcal{M}(\mathfrak{h}(\mathcal{P}))$ for $\mathfrak{h}(\mathcal{P})$ is isomorphic to the quotient model $\overline{\mathcal{M}(\mathcal{P})}$ for $\mathcal{P}$ [6]. Thus the state-space reduction potentially available by model checking $\overline{\mathcal{M}(\mathcal{P})}$ can be realised by instead model checking $\mathcal{M}(\mathfrak{h}(\mathcal{P}))$. Theoretical and experimental results show that for systems consisting of many processes, where each process has a small local state-space, the BDD representation of $\mathcal{M}(\mathfrak{h}(\mathcal{P}))$ can be significantly smaller than the BDD for $\mathcal{M}(\mathcal{P})$ or $\overline{\mathcal{M}(\mathcal{P})}$. The approach is extended to provide symmetry reduction for MDPs in [7].

As discussed in Section I, a major drawback to current generic representatives techniques is the fact that specifications must be expressed in an extremely restricted input language before translation to generic form can be performed. Here we significantly extend the approach to work with a richer input language supporting multiple families of symmetric processes. The state of each process is determined by the values of multiple local variables, and sophisticated process behaviour can be specified via arithmetic and boolean expressions over local and global variables.

\section{Symmetric Probabilistic SpecificAtion LANGUAGE}

We define a simple but general language for specifying symmetric, probabilistic systems, which we call Symmetric Probabilistic Specification Language (SPSL). This is loosely based on Symmetric PRISM [7], a subset of the model specification language for PRISM [9]. The connection with PRISM is beneficial for several reasons. Firstly, other tools make direct use of the PRISM modelling language or accept inputs that can be generated by PRISM. Secondly, translations have been developed to the PRISM language from a variety of specification formalisms (including probabilistic variants of several process calculi).

\section{A. Syntax}

The syntax for SPSL is presented via a BNF-like grammar in Fig. 1. Note that $\bowtie$ is a binary operator in $\{+,-, *, /, * *,<, \leq,>, \geq,=, \neq, \wedge, \vee\}$, where $* *$ denotes exponentiation; $\bigcirc$ is an operator in $\left\{\sum, \Pi, \bigwedge, \bigvee\right\}$; number denotes a numeric literal; name is the name of a variable, and local-name/global-name is the name of a local/global variable specifically.

An SPSL specification consists of an optional set of global variable declarations followed by a set of module type declarations, each representing a particular class of symmetric processes.

A variable declaration consists of a name, type and initial value. For simplicity, we use a basic type system in which variables are either booleans or finite integer ranges. The domain and initial value of a variable $v$ are denoted by $\operatorname{dom}(v)$ and $\operatorname{init}(v)$, respectively.

A module type declaration consists of a name $(M)$, an integer specifying the number of running instances of $M$ (denoted $\# M$ ), a set of local variable declarations and a set of statements. The set of all module type names is denoted $\mathbf{M}$ and we refer to the running instances of $M \in \mathbf{M}$ as $M_{1}, M_{2}, \ldots, M_{\# M}$.

The set of global variables is denoted global and the variables local to module type $M$ are denoted $\operatorname{var}(M)$. For simplicity, but without loss of generality, we assume that all variable names and module type names in a specification are distinct. For variable $v \in \operatorname{var}(M)$, we write $v_{i}$ for the corresponding local variable of module instance $M_{i}$. We use $\operatorname{var}_{t}(M)$ to denote the $t$-th variable in the specification of $M$, and $\operatorname{var}_{t}(M)_{i}$ the copy belonging to $M_{i}$.

The behaviour of instances of module type $M$ is given by a set of statements defined with respect to a symbolic module instance $M_{i}$; the behaviour of a concrete instance $M_{z}$ (for any $1 \leq z \leq \# M$ ) is derived from these statements by replacing each symbolic variable $v_{i}$ with the corresponding concrete variable $v_{z}$. Statements are guarded commands: the guard is an expression determining whether a statement is executable in a given state; the effect of the statement is defined by a stochastic update, which takes the form $e^{1}: u^{1}+e^{2}: u^{2}+\ldots+e^{k}: u^{k}$ where the $e^{j}$ are expressions (giving probability) and the $u^{j}$ updates. Each update $u^{j}$ is either a no-op (skip), or a set of concurrent updates (denoted by (1) to distinct variables that are either global or local to $M$.

Guards, probabilities and updates to variables in module type $M$ are given by expressions derived from the production rule expr $\left(M_{i}\right)$. This is defined in terms of two other rules: loc-expr $(M)$, which permits expressions over variables from $\operatorname{var}(M)$, and symm-expr, which defines fully symmetric expressions composed of globals, constants and combinations of a local expression instantiated over all modules of a given type. Thus $\operatorname{expr}\left(M_{i}\right)$ defines expressions which are symmetric with respect to $M_{i}$. These are composed of fully symmetric expressions, combinations of a local expression 


\begin{tabular}{|c|c|c|}
\hline specification & $::=$ & global-variables $^{?}$ module $^{+}$ \\
\hline global-variables & $::=$ & globals $\left\{\right.$ var-decl $\left.^{+}\right\}$ \\
\hline module & $::=$ & 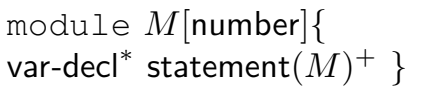 \\
\hline var-decl & $::=$ & name : type init constant \\
\hline type & $::=$ & [number..number] | bool \\
\hline constant & $::=$ & true | false | number \\
\hline statement $(M)$ & $::=$ & $\operatorname{expr}\left(M_{i}\right) \rightarrow$ st-update $(M)$ \\
\hline st-update $(M)$ & $::=$ & $\begin{array}{l}\operatorname{expr}\left(M_{i}\right): \text { update }(M)+\ldots \\
+\operatorname{expr}\left(M_{i}\right): \text { update }(M)\end{array}$ \\
\hline $\operatorname{update}(M)$ & $::=$ & $\begin{array}{l}\text { skip } \mid\left(\text { name }:=\operatorname{expr}\left(M_{i}\right)\right) \\
\|\ldots\|\left(\text { name }:=\operatorname{expr}\left(M_{i}\right)\right)\end{array}$ \\
\hline symm-expr & $::=$ & $\begin{array}{l}\text { constant | global-name } \\
\bigcirc_{1 \leq j \leq \# N} \text { loc-expr }(N)_{j} \\
\text { (for some module type } N \text { ) } \\
\text { symm-expr } \bowtie \text { symm-expr } \\
\neg \text { symm-expr | (symm-expr) }\end{array}$ \\
\hline loc-expr $(M)$ & $\begin{array}{l}:= \\
\mid \\
\mid\end{array}$ & $\begin{array}{l}\text { constant | local-name } \\
\text { loc-expr }(M) \bowtie \text { loc-expr }(M) \\
\neg \text { loc-expr }(M) \\
(\text { loc-expr }(M))\end{array}$ \\
\hline $\operatorname{expr}\left(M_{i}\right)$ & $::=$ & $\begin{array}{l}\text { loc-expr }(M)_{i} \quad \text { symm-expr } \\
\bigcirc_{1 \leq j \neq i \leq \# M} \text { loc-expr }(M)_{j} \\
\operatorname{expr}\left(M_{i}\right) \bowtie \operatorname{expr}\left(M_{i}\right)\end{array}$ \\
\hline & $\mid$ & $\neg \operatorname{expr}\left(M_{i}\right) \quad \mid\left(\operatorname{expr}\left(M_{i}\right)\right)$ \\
\hline
\end{tabular}

Figure 1. Syntax of Symmetric Probabilistic Specification Language (SPSL).

instantiated over all modules of type $M$ except $M_{i}$ and local expressions of the form loc-expr $(M)_{i}$.

The symmetric conjunctions and disjunctions of SPSL generalise simple symmetric boolean expressions provided by previous approaches [5], [6]. Additionally, SPSL allows symmetric summations and products, and allows expressions to operate on a mixture of local and global variables. This affords a great deal more flexibility when modelling.

\section{B. MDP semantics}

We now define semantics for an SPSL specification $\mathcal{P}$ in terms of an MDP $\mathcal{M}(\mathcal{P})=\left(S, s_{0}\right.$, Steps $)$. For brevity, we do not consider errors such as division-by-zero or probability distributions which do not sum to one; it is easy to extend the semantics to handle this via a designated error state.

The local state-space for a single module $M_{i}$ of type $M$ is $S\left(M_{i}\right)=\bigotimes_{v \in \operatorname{var}(M)} \operatorname{dom}(v)$ and the state-space for the global variables of $\mathcal{P}$ is $G=\bigotimes_{v \in \text { global }} \operatorname{dom}(v)$. The global state-space of $\mathcal{M}(\mathcal{P})$ is:

$$
S=G \times\left(\bigotimes_{M \in \mathrm{M}}\left(\bigotimes_{1 \leq i \leq \# M} S\left(M_{i}\right)\right)\right)
$$

Let $e$ be an expression of the form expr $\left(M_{i}\right)$, and $s$ a (local or global) state. Then $\operatorname{eval}(s, e)$ is the result of evaluating the expression $e$ at $s$. If $e$ is a boolean expression, we use $s \models e$ as shorthand for $\operatorname{eval}(s, e)=$ true.

Let $s \in S$ and $u$ an update of the form $\left(v^{1}:=e^{1}\right) \|$ $\left(v^{2}:=e^{2}\right)\|\ldots\|\left(v^{k}:=e^{k}\right)$, where the $v^{j}$ are distinct variables. Then $\operatorname{exec}(s, u)$ is the state identical to $s$ except that the value of each variable $v_{j}$ is equal to $\operatorname{eval}\left(s, e^{j}\right)$. We also define $\operatorname{exec}(s$, skip $)=s$.

We now define the probabilistic transition function Steps. Let $s \in S, M \in \mathbf{M}$ and $1 \leq z \leq \# M$. Let $e_{z} \rightarrow u_{z}$ be a concrete statement of module $M_{z}$. Suppose $u_{z}$ has the form $e^{1}: u^{1}+e^{2}: u^{2}+\ldots+e^{k}: u^{k}$. If $s=e_{z}$ then $\mu \in \operatorname{Steps}(s)$, where $\mu: S \rightarrow[0,1]$ is defined as follows: for $t \in S$, $\mu(t)=\sum_{1 \leq j \leq k, \operatorname{exec}\left(s, u^{j}\right)=t} \operatorname{eval}\left(s, e^{j}\right)$.

\section{Symmetric PCTL}

Let $\mathcal{P}$ be an SPSL specification, and $A$ the set of all expressions of the form symm-expr defined over the variables of $\mathcal{P}$. Taking $A$ to be our set of atoms, we refer to the set of $P C T L$ formulae over $A$ as symmetric PCTL (SPCTL). The $S P C T L$ logic allows us to reason about properties of SPSL specifications which do not distinguish between individual modules within a given module type.

\section{Running example}

To illustrate the SPSL language and associated techniques, we introduce a running example: a simple SPSL specification, describing 3 master processes and a pool of 10 worker processes. A master probabilistically issues requests to the workers via a shared channel m_to_w, modelled abstractly as a counter, becoming inactive only when no worker processes remain active. A worker process continuously removes requests from the shared channel by decrementing the counter. When not processing work, a worker process may go to sleep with probability 0.1 .

Fig. 2 shows the master/worker SPSL specification. The labels (1) to (7) are used for explanation in Section IV-C and should otherwise be ignored.

The following $S P C T L$ property states that the probability of reaching a state where all workers are asleep and there are outstanding requests on the shared channel is less than 0.01: $P_{<0.01}\left[\diamond\left(\mathrm{m}_{\text {_to_w }}>0 \wedge \bigwedge_{1 \leq j \leq 10}\right.\right.$ awake $\left.\left._{j}=0\right)\right]$.

\section{E. SPSL Specifications are Symmetric}

SPSL has been designed to guarantee full symmetry between instances of each module type. Formally, this can be stated as follows. For a module type $M$ of $\mathcal{P}$, let $\operatorname{Sym}(M)$ denote the group of permutations of the set $\left\{M_{1}, M_{2}, \ldots, M_{\# M}\right\}$. Elements of $\operatorname{Sym}(M)$ act on a state $s$ of $\mathcal{M}(\mathcal{P})$ in the obvious way. Suppose that $\mathbf{M}=$ $\left\{M^{1}, M^{2}, \ldots, M^{d}\right\}$. Then $\operatorname{Sym}(\mathbf{M})=\left\{\alpha^{1} \alpha^{2} \ldots \alpha^{d}: \alpha^{j} \in\right.$ $\left.\operatorname{Sym}\left(M^{j}\right)(1 \leq j \leq d)\right\}$ is the largest group of module permutations that preserves module types. The fully symmetric 


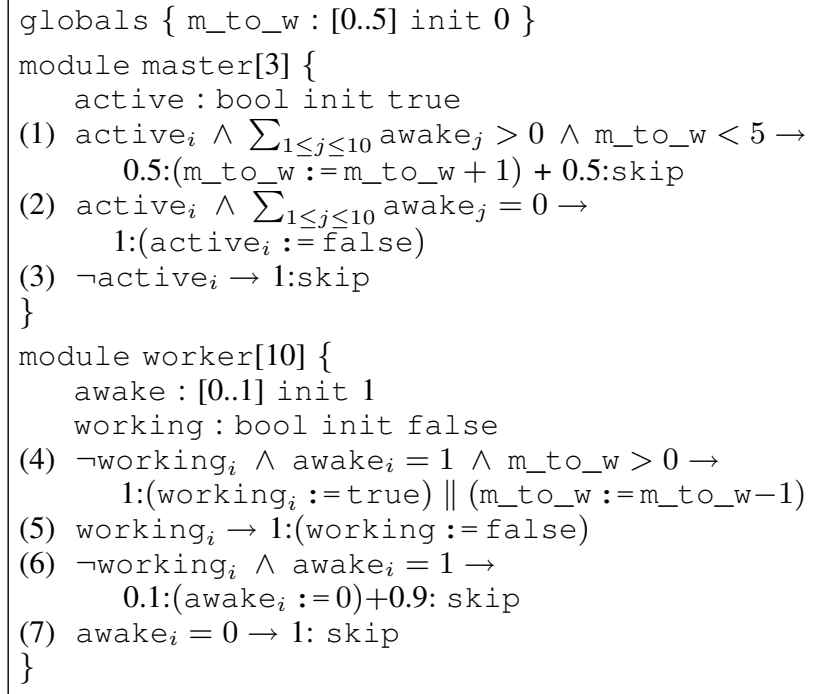

Figure 2. Example SPSL specification for the master/worker system described in Section III-D.

syntax of SPSL guarantees that every element of $\operatorname{Sym}(\mathbf{M})$ is an automorphism of $\mathcal{M}(\mathcal{P})$, and any $S P C T L$ formula is symmetric w.r.t. $\operatorname{Sym}(\mathbf{M})$. This, with Theorem 2, leads to the following result:

Theorem 3: Let $\overline{\mathcal{M}(\mathcal{P})}$ denote the quotient MDP for $\mathcal{M}(\mathcal{P})$ w.r.t. $\operatorname{Sym}(\mathbf{M})$ and let $\phi$ be an $S P C T L$ formula. Then $\mathcal{M}(\mathcal{P}) \models \phi \Leftrightarrow \overline{\mathcal{M}(\mathcal{P})} \models \phi$.

Suppose the module types for $\mathcal{P}$ are $M^{1}, M^{2}, \ldots, M^{d}$ for some $d>0$. It is easy to show that $|\operatorname{Sym}(\mathbf{M})|=$ $\left|\# M^{1}\right| ! \times\left|\# M^{2}\right| ! \times \cdots \times\left|\# M^{d}\right| !$. Since the extent of symmetry reduction depends on the size of the symmetry group (see Section II), there is potential for a large reduction factor with multiple instances of each module type.

\section{TRAnslation to GEnERIC Form}

We now show how an SPSL specification $\mathcal{P}$ together with an $S P C T L$ property $\phi$ can be translated into a generic specification $\mathfrak{h}(\mathcal{P})$ and property $\mathfrak{h}(\phi)$, such that $\mathcal{M}(\mathcal{P}) \models$ $\phi \Leftrightarrow \mathcal{M}(\mathfrak{h}(\mathcal{P})) \mid=\mathfrak{h}(\phi)$.

In this section, we impose a restriction on $\mathcal{P}$, requiring that in a an update $v_{i}:=e$ to a local variable, expression $e$ contains only local variables and constants.

\section{A. The translation algorithm}

Fig. 3 presents a set of syntax-directed translation rules, based on the SPSL grammar of Fig. 1, for translation of an SPSL specification $\mathcal{P}$ into a generic SPSL specification, $\mathfrak{h}(\mathcal{P})$. We call $\mathfrak{h}$ the translation function.

We introduce some additional notation. Let $S(M)$ denote the local state-space for some module type $M$ (which is the same for any instance of $M$ ) and let $|S(M)|=t$. Using the natural lexicographic ordering over tuples in $S(M)$, we define a bijection $f_{M}: S(M) \rightarrow\{1,2, \ldots, t\}$. For an expression $e, \operatorname{SAT}_{M}(e)=\{l \in S(M): l \models e\}$ denotes the subset of local states of $M$ in which $e$ holds.

The first two rules in Fig. 3 state that the global variables of $\mathfrak{h}(\mathcal{P})$ are the same as for $\mathcal{P}$ and that, for every module type declaration $M$ in $\mathcal{P}, \mathfrak{h}(\mathcal{P})$ contains a single instance of a module of type generic_ $M$. This module has $|S(M)|$ counter variables with range $0, \ldots, \# M$. Each variable count $\_M_{\_} j$ records the number of instances of $M$ residing in state $f_{M}^{-1}(j)$ (for $1 \leq j \leq t$ ). We initialise count_M_ $f_{M}(\operatorname{init}(M))$ to $\# M$ and all others to 0 .

When translating statements for a module type declaration $M$, we must consider the fact that these statements comprise not only symmetric expressions, but also expressions referring to the local state of $M$ (i.e. those from the grammar production loc-expr( $M)$ in Fig. 1). For the purposes of translation, we assume that the guard of a statement is of the form $e_{i} \wedge \operatorname{expr}\left(M_{i}\right)$, where $e$ has the form loc-expr $(M)$. We then split the translation into cases, one for each $l \in \operatorname{SAT}_{M}(e)$. This loses no generality since, in the worst case, a guard of the general form $\operatorname{expr}\left(M_{i}\right)$ is trivially of the form given above, by taking $e_{i}$ to be true. In this case $\left|\mathrm{SAT}_{M}(e)\right|=|S(M)|$, i.e. each local state of $M$ is treated separately, meaning that the worst case complexity for our translation algorithm is $O\left(|\mathcal{P}| \times \max _{M \in \mathbf{M}}|S(M)|\right)$ where $|\mathcal{P}|$ is the number of statements in $\mathcal{P}$. In practice, guardedcommand style descriptions of multi-process systems are very often written with guards of the form $e_{i} \wedge \operatorname{expr}\left(M_{i}\right)$ where $e_{i}$ is reasonably strong, so the worst case complexity is rarely realised.

Given a statement $x$ of the form $e_{i} \wedge \operatorname{expr}\left(M_{i}\right) \rightarrow$ stoch-update $(M)$, we generate, for each $l \in \operatorname{SAT}_{M}(e)$ a separate generic statement corresponding to the original statement $x$ as follows. The $e_{i}$ part of the guard is translated into the condition count_ $M_{-} f_{M}(l)>0$. This condition asserts that some instance of $M$ has local state $l$ and thus the local part of the guard is satisfied for this module. The remainder of the guard, of the form expr $\left(M_{i}\right)$, and the stochastic update stoch-update $(M)$, are translated in the context of $l$ using the rules $\mathfrak{h}\left(\operatorname{expr}\left(M_{i}\right), l\right)$ and $\mathfrak{h}($ stoch-update $(M), l)$.

The most intricate part of the translation process is the translation of variable updates. Consider an update:

$$
\left(v_{i}^{1}:=e_{i}^{1}\right)\|\ldots\|\left(v_{i}^{t}:=e_{i}^{t}\right)\left\|\left(g^{1}:=d^{1}\right)\right\| \ldots \|\left(g^{r}:=d^{r}\right)
$$

where $v^{j} \in \operatorname{var}(M), e^{j}$ (according to our restriction) has the form loc-expr $(M)(1 \leq j \leq t), g^{j} \in$ global and $d^{j}$ has the form $\operatorname{expr}\left(M_{i}\right)(1 \leq j \leq r)$.

Let $l^{\prime}$ be the local state reached by executing all local variable updates (which can be statically computed). The update $\mathfrak{h}(u, l)$ (see Fig. 3) has the effect of: decrementing count_M_ $f_{M}(l)$ (representing a process leaving state $l$ ), incrementing count_$M \_f_{M}\left(l^{\prime}\right)$ (representing this process entering state $l^{\prime}$ ), and updating the value of each global variable $g^{j}$ according to the generic expression $\mathfrak{h}\left(d^{k}, l\right)$ 


\begin{tabular}{|c|c|}
\hline $\mathcal{P}$ & $\mathfrak{h}(\mathcal{P})$ \\
\hline global-variables module ... module & global-variables $\mathfrak{h}($ module $) \ldots \mathfrak{h}($ module $)$ \\
\hline module & 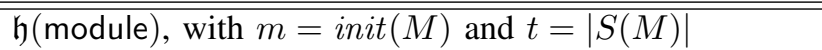 \\
\hline $\begin{array}{l}\text { module } M[k]\{ \\
\quad \text { var-decl* } \\
\text { statement }(M) \ldots \text { statement }(M) \\
\}\end{array}$ & $\begin{array}{l}\text { module generic_ } M[1]\{ \\
\quad \text { count_M_1: }[0 . . k] \text { init } 0 \\
\quad \ldots \text { count_M_ } f_{M}(m):[0 . . k] \text { init } k \ldots \\
\quad \text { count_M_t }:[0 . . k] \text { init } 0 \\
\\
\mathfrak{h}(\text { statement }(M)) \ldots h(\text { statement }(M)) \\
\}\end{array}$ \\
\hline statement $(M)$, where $e$ has form local-expr $(M)$ & $\overline{\mathrm{h}}($ statement $(M))$, with $\mathrm{SAT}_{M}(e)=\left\{l^{1}, \ldots, l^{z}\right\}$ \\
\hline $\begin{aligned} e_{i} & \wedge \operatorname{expr}\left(M_{i}\right) \\
& \rightarrow \text { stoch-update }(M)\end{aligned}$ & $\begin{array}{l}\text { count_M_f } f_{M}\left(l^{1}\right)>0 \wedge \mathfrak{h}\left(\operatorname{expr}\left(M_{i}\right), l^{1}\right) \\
\quad \rightarrow \mathfrak{h}\left(\operatorname{stoch}-u p d a t e(M), l^{1}\right) \ldots \\
\text { count_M_f } f_{M}\left(l^{z}\right)>0 \wedge \mathfrak{h}\left(\operatorname{expr}\left(M_{i}\right), l^{z}\right) \\
\quad \rightarrow \mathfrak{h}\left(\text { stoch-update }(M), l^{z}\right)\end{array}$ \\
\hline stoch-update $(M)$ & $\mathfrak{h}$ h(stoch-update $(M), l)$ \\
\hline $\begin{array}{l}\operatorname{expr}\left(M_{i}\right): \text { update }(M)+\ldots \\
\quad+\operatorname{expr}\left(M_{i}\right): \text { update }(M)\end{array}$ & $\begin{array}{l}\mathfrak{h}\left(\operatorname{expr}\left(M_{i}\right), l\right): \mathfrak{h}(\text { update }(M), l)+\ldots \\
\quad+\mathfrak{h}\left(\operatorname{expr}\left(M_{i}\right), l\right): \mathfrak{h}(\text { update }(M), l)\end{array}$ \\
\hline $\begin{array}{l}\text { update }(M) \text {, where } v^{j} \in \operatorname{var}(M), g^{j} \in \text { global } \\
\text { and } e^{j} / d^{j} \text { has form local-expr }(M) / \operatorname{expr}\left(M_{i}\right)\end{array}$ & $\begin{array}{l}\mathfrak{h}(\text { update }(M), l), \text { where } \\
l^{\prime}=l\left[v^{1}:=\operatorname{eval}\left(l, e^{1}\right), \ldots, v^{t}:=\operatorname{eval}\left(l, e^{t}\right)\right]\end{array}$ \\
\hline $\begin{array}{l}\text { skip } \\
\left(v_{i}^{1}:=e_{i}^{1}\right)\|\ldots\|\left(v_{i}^{t}:=e_{i}^{t}\right) \\
\quad\left\|\left(g^{1}:=d^{1}\right)\right\| \ldots \|\left(g^{r}:=d^{r}\right)\end{array}$ & $\begin{array}{l}\text { skip } \\
\left(\text { count_M_ } f_{M}(l):=\text { count_M_} f_{M}(l)-1\right) \\
\quad \|\left(\text { count_M_ } f_{M}\left(l^{\prime}\right):=\text { count_M_ } f_{M}\left(l^{\prime}\right)+1\right) \\
\quad\left\|\left(g^{1}:=\mathfrak{h}\left(d^{1}, l\right)\right)\right\| \ldots \|\left(g^{r}:=\mathfrak{h}\left(d^{r}, 1\right)\right)\end{array}$ \\
\hline expr $\left(M_{i}\right)$, where $e$ has form local-expr $(M)$ & $\overline{\mathfrak{h}}\left(\operatorname{expr}\left(M_{i}\right), l\right)$ \\
\hline $\begin{array}{l}\text { e } \\
\text { symm-expr } \\
\sum_{1 \leq j \neq i \leq \# M} e_{j} \\
\prod_{1 \leq j \neq i \leq \# M} e_{j} \\
\bigwedge_{1 \leq j \neq i \leq \# M} e_{j} \\
\bigvee_{1 \leq j \neq i \leq \# M} e_{j} \\
\operatorname{expr}\left(M_{i}\right) \bowtie \operatorname{expr}\left(M_{i}\right) \\
\neg \operatorname{expr}\left(M_{i}\right) \\
\left(\operatorname{expr}\left(M_{i}\right)\right)\end{array}$ & 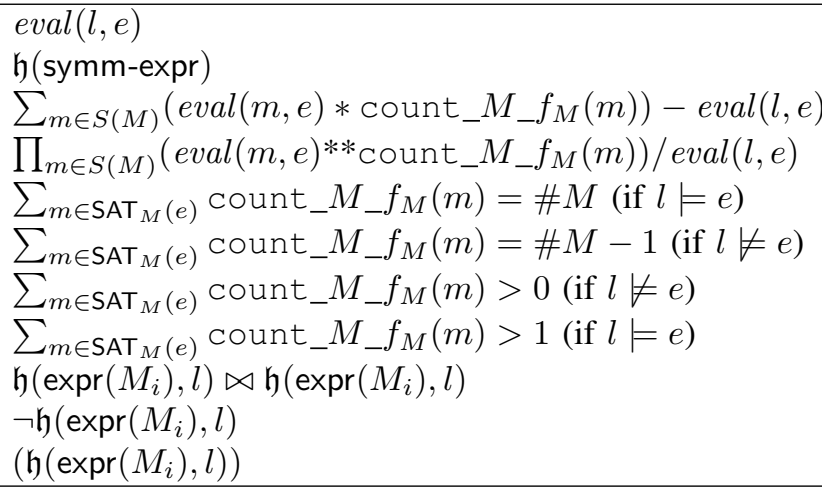 \\
\hline symm-expr, where $e$ has form local-expr $(N)$ & (symm-expr) \\
\hline $\begin{array}{l}\text { constant } \\
\text { name (where name is a global variable) } \\
\sum_{1 \leq j \leq \# N} e_{j} \\
\prod_{1 \leq j \leq \# N} e_{j} \\
\bigwedge_{1 \leq j \leq \# N} e_{j} \\
\bigvee_{1 \leq j \leq \# N} e_{j} \\
\text { symm-expr } \bowtie \text { symm-expr } \\
\neg \text { symm-expr } \\
\text { (symm-expr) }\end{array}$ & 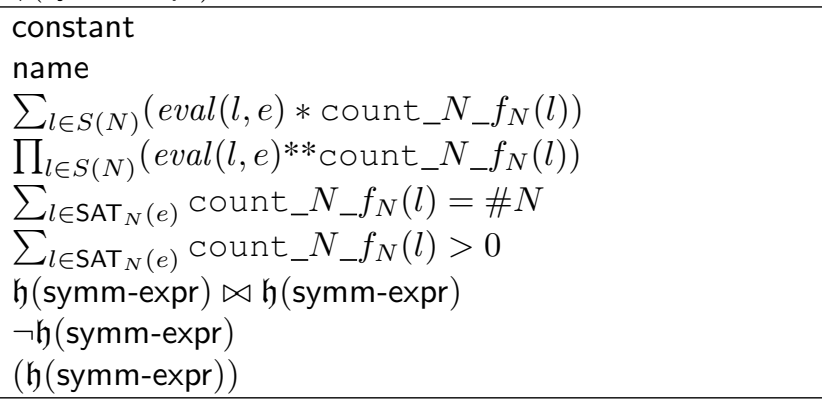 \\
\hline
\end{tabular}

Figure 3. Rules for translating an SPSL specification $\mathcal{P}$ to a generic form $\mathfrak{h}(\mathcal{P})$. 
$(1 \leq j \leq r)$.

The remaining rules in Fig. 3 are concerned with translation of expressions of the forms $\operatorname{expr}\left(M_{i}\right)$ and symm-expr. The rationale for these rules is similar to the rationale presented in [6], generalised to our more expressive language, and extended with symmetric summations and products.

\section{B. Model checking $\mathcal{M}(\mathcal{P})$ via $\mathcal{M}(\mathfrak{h}(\mathcal{P}))$}

Let $\phi$ be an SPCTL property over $\mathcal{P}$. Recall from Section III-C that the atoms of $\phi$ are expressions of the form symm-expr over the variables of $\mathcal{P}$. We translate $\phi$ into a generic $S P C T L$ property $\mathfrak{h}(\phi)$ over $\mathfrak{h}(\mathcal{P})$ by replacing each atom $e$ appearing in $\phi$ with $\mathfrak{h}(e)$, using the translation rules for symm-expr in Fig. 3.

Let $\mathcal{P}$ be an SPSL specification with associated MDP $\mathcal{M}(\mathcal{P})=\left(S, s_{0}\right.$, Steps $)$. Since $\mathfrak{h}(\mathcal{P})$ is itself an SPSL specification, the MDP $\mathcal{M}(\mathfrak{h}(\mathcal{P}))=\left(S^{\prime}, s_{0}^{\prime}\right.$, Steps $\left.{ }^{\prime}\right)$ associated with $\mathfrak{h}(\mathcal{P})$ can be derived directly as shown in Section III-B. However, it is useful to describe the states of $\mathcal{M}(\mathfrak{h}(\mathcal{P}))$ with reference to $\mathcal{P}$. If $\mathbf{M}$ is the set of module types for $\mathcal{P}$, the state set $S^{\prime}$ associated with $\mathcal{M}(\mathfrak{h}(\mathcal{P}))$ is:

$$
S^{\prime}=G \times\left(\bigotimes_{M \in \mathrm{M}} S(\text { generic_ } M)\right) .
$$

We define a function $\delta: S \rightarrow S^{\prime}$ that maps equivalent states in $S$ to a single state in $S^{\prime}-$ a generic representative.

For $M \in \mathbf{M}, s \in S$ and $l \in S(M), \delta_{M}^{l}(s)$ denotes the number of instances of $M$ with local state $l$ at $s$. Thus $\delta_{M}^{l}$ maps $S$ to $\{0,1, \ldots, \# M\}$. Suppose that $S(M)=\left\{l_{1}<l_{2}<\cdots<l_{t}\right\}$ for some $t \geq 0$. Let $\delta_{M}(s)=\left(\delta_{M}^{l_{1}}(s), \delta_{M}^{l_{2}}(s), \ldots, \delta_{M}^{l_{t}}(s)\right)$. The mapping $\delta_{M}: S \rightarrow S$ (generic_ $M$ ) transforms $s$ into a tuple counting the number of instances of $M$ residing in each local state of $S(M)$.

Suppose that the module types for $\mathcal{P}$ are, for some $d>0$, $M^{1}, M^{2}, \ldots, M^{d}$, and let $s=\left(s_{\text {global }}, s_{\text {local }}\right) \in S$. Then we define $\delta(s)=\left(s_{\text {global }}, \delta_{M^{1}}(s), \delta_{M^{2}}(s), \ldots, \delta_{M^{d}}(s)\right)$. We therefore have $\delta(s)_{\text {global }}=s_{\text {global }}$ and $\delta(s)_{\text {local }}=$ $\left(\delta_{M^{1}}(s), \delta_{M^{2}}(s), \ldots, \delta_{M^{d}}(s)\right)$.

We use the function $\delta$ to give a list of properties of our translation method (see [14] for proofs).

1) If $e$ has the form symm-expr then $\operatorname{eval}(s, e)=$ $\operatorname{eval}(\delta(s), \mathfrak{h}(e))$. If $e$ is boolean then $s \models e \Leftrightarrow \delta(s) \models$ $\mathfrak{h}(e)$.

2) If $e$ has the form $\operatorname{expr}\left(M_{i}\right)$ and $M_{i}$ has local state $l$ at $s$ then $\operatorname{eval}(s, e)=\operatorname{eval}(\delta(s), \mathfrak{h}(e, l))$. If $e$ is boolean then $s \models e \Leftrightarrow \delta(s) \models \mathfrak{h}(e, l)$.

3) If $u$ has the form update $(M)$ and $M_{i}$ has local state $l$ at $s$ then $\operatorname{exec}(\delta(s), \mathfrak{h}(u, l))=\delta(\operatorname{exec}(s, u))$.

The above results allow us to prove that the reachable parts of $\overline{\mathcal{M}(\mathcal{P})}$ and $\mathcal{M}(\mathfrak{h}(\mathcal{P}))$ are isomorphic, and that we can infer $S P C T L$ properties of $\mathcal{M}(\mathcal{P})$ by checking $\mathcal{M}(\mathfrak{h}(\mathcal{P}))$. (See [14] for proofs.)
Without loss of generality, in the following theorems we consider MDPs to be restricted to reachable states.

Theorem 4: Let $\mathcal{P}$ be an SPSL specification. Let $\overline{\mathcal{M}(\mathcal{P})}=\left(\bar{S}, \overline{s_{0}}, \overline{\text { Steps }}\right)$ be the quotient MDP for $\mathcal{M}(\mathcal{P})=$ $\left(S, s_{0}\right.$, Steps $)$ w.r.t. the group $\operatorname{Sym}(\mathbf{M})$. Then $\delta$, restricted to $\bar{S}$, is an isomorphism from $\overline{\mathcal{M}(\mathcal{P})}$ to $\mathcal{M}(\mathfrak{h}(\mathcal{P}))$.

Theorem 1 now applies, in which $\gamma$ is taken to be the translation function $\mathfrak{h}$, which is indeed a bijection over the atoms of SPCTL, i.e. expressions of the form symm-expr.

Theorem 5: $\mathcal{M}(\mathcal{P}) \models \phi \Leftrightarrow \mathcal{M}(\mathfrak{h}(\mathcal{P})) \models \mathfrak{h}(\phi)$.

Two extensions to our approach that have been implemented and deserve note are: the relaxation of the restriction to variable updates described above to allow updates to local variables with expressions involving globals; and the extension of the approach to several other model types (Kripke structions, Discrete Time Markov Chains and Continuous Time Markov Chains). We do not include details here for space reasons.

\section{Running example}

Fig. 4 shows the generic version of the running example of Fig. 2. Counter variables count_master $j$ and count_worker $\_j$ are abbreviated to $\mathrm{m}_{-} j$ and $\mathrm{w} \_j$, respectively. There are two counter variables for the generic master process, since there are two possible master local states, $(T)$ and $(F)$ depending whether boolean local variable active is true or false. Similarly, there are four worker local states, $(0, F),(0, T),(1, F),(1, T)$, corresponding to the possible configurations of the awake and working local variables. For each statement labelled (1) to (7) in Fig. 2, Fig. 4 contains a label associated with corresponding statements in the reduced specification. We describe the translation process for statements (1) and (5).

Consider statement (1) of Fig. 2. The guard for this statement has the form $e_{i} \wedge d$ where $e$ is active and $d$ is $\sum_{1 \leq j \leq 10}$ awake $_{j}>0$. We statically compute $\mathrm{SAT}_{\text {master }}($ active $)=\{(T)\}$, where $(T)=l$, say. We have $f_{\text {master }}(l)=2$, so we output a single generic statement with guard $\mathrm{m} \_2>0 \wedge \mathfrak{h}(d, l)$. According to the translation rules of Fig. $3, \mathfrak{h}(d, l)$ reduces to $\mathfrak{h}\left(d^{\prime}\right)>0$, where $d^{\prime}$ is the sum of the expression awake over all worker modules. The translated expression $\mathfrak{h}\left(d^{\prime}\right)$ is a linear combination over the counter variables representing worker local states. For worker local state $m$, the linear combination includes the term $\operatorname{eval}(m$, awake $) * w_{-} f_{\text {worker }}(m)$. This is the contribution to the original sum for every worker module in state $m$. Thus for $m=(0, F)$ we output the term $0 * \mathrm{w}_{-} 1$ because $\operatorname{eval}(m$, awake $)=0$ and $f_{\text {worker }}(m)=1$. For $m=(1, T)$ we have $f_{\text {worker }}(m)=4$ and $\operatorname{eval}(m$, awake $)=1$, resulting in the term $1 * w_{-} \_4$. The complete generic guard is shown in statement (1) of Fig. 4. (A constant folding optimization can be used to simplify this guard by removing 


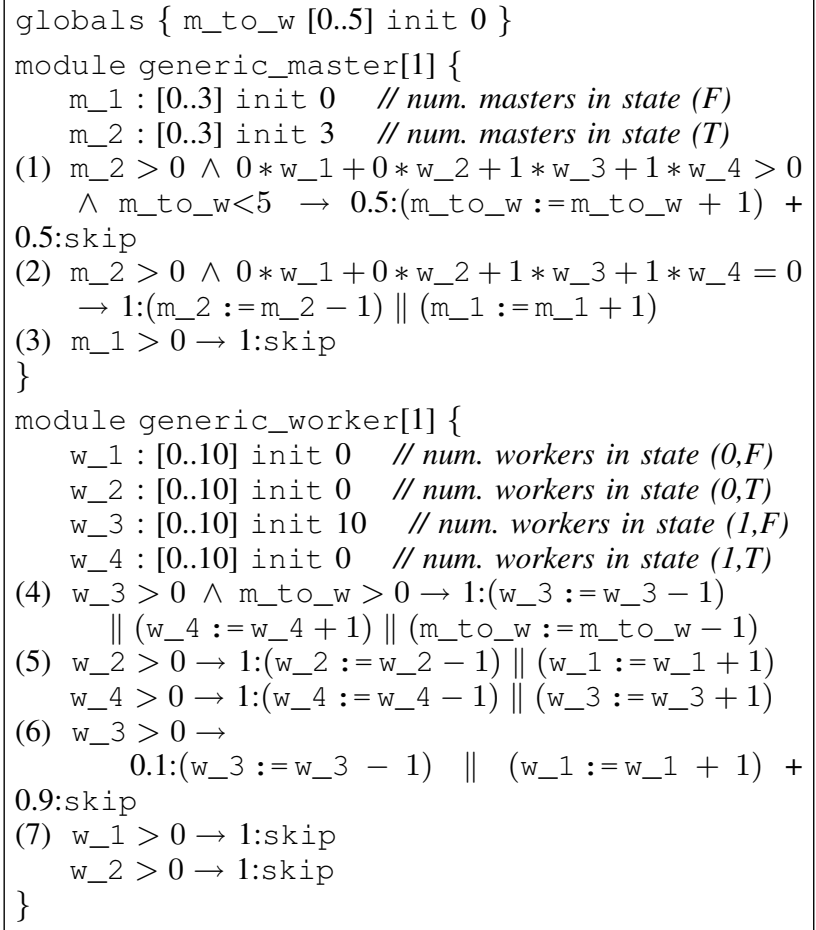

Figure 4. Generic SPSL specification corresponding to the master/worker specification of Fig. 2.

redundant multiplications by zero and one.) The stochastic update associated with the reduced statement is the same as for the original, since the update does not modify local state. Note that the probability distribution is passed through unchanged.

The guard for statement (5) in Fig. 2, for a worker module, can be re-written as working $i \wedge$ true, which has the form local-expr(worker $)_{i} \wedge \operatorname{expr}\left(\right.$ worker $\left._{i}\right)$. We compute $\mathrm{SAT}_{\text {worker }}($ working $)=\{(0, T),(1, T)\}$ and generate a generic statement for each of these local states. Considering the second local state, $(1, T)=l$ say, we have $f_{\text {worker }}(l)=$ 4 , so the generic guard is $\mathrm{w}_{-} 4>0 \wedge$ true, or just $\mathrm{w}_{-} 4>0$. The stochastic update associated with the statement consists of one local update, working $i:=0$. Given that the worker module executing this update is in local state $l=(1, T)$, after the update the worker has local state $m=(1, F)$. Since $f_{\text {worker }}(l)=4$ and $f_{\text {worker }}(m)=3$, the generic update involves decrementing $\mathrm{w}_{-} 4$ and incrementing W_3.

Recall the SPCTL property for the master/worker specification presented at the end of Section III-D. We convert this property to generic form by applying the translation function $\mathfrak{h}$ to each subformula of the form symm-expr. Subformula m_to_w $>0$ is left unchanged by $\mathfrak{h}$. Continuing to use the abbreviations $\mathrm{m}_{-} j$ and $\mathrm{w}_{\_} j$, we have:

$$
\mathfrak{h}\left(\bigwedge_{1 \leq j \leq 10} \text { awake }_{j}=0\right)
$$

$$
\begin{aligned}
& =\left(\sum_{l \in \mathrm{SAT}_{\text {worker }}(\text { awake }=0)} \mathrm{W}_{\text {_ }} f_{\text {worker }}(l)=10\right) \\
& =\left(\sum_{l \in\{(0, F),(0, T)\}} \mathrm{W}_{-} f_{\text {worker }}(l)=10\right) \\
& =\left(\mathrm{w}_{\text {_ }} f_{\text {worker }}((0, F))+\mathrm{w}_{\text {_orker }}((0, T))=10\right) \\
& =\left(\mathrm{w} \_1+\mathrm{w} \_2=10\right)
\end{aligned}
$$

Thus the generic $S P C T L$ formula is:

$$
P_{<0.01}\left[\diamond\left(\mathrm{m} \_ \text {to_w }>0 \wedge \mathrm{w}_{-} 1+\mathrm{w}_{-} 2=10\right)\right] \text {. }
$$

\section{EXPERIMENTAL RESUlts}

We have implemented our techniques in a tool, GRIP (Generic Representatives in PRISM). It accepts a PRISM specification conforming to a syntax analogous to SPSL, and outputs a generic specification for model checking with PRISM. GRIP supports MDP, DTMC and CTMC specifications, with associated symmetric temporal properties.

We apply GRIP to seven case studies: Aspnes \& Herlihy's randomised consensus protocol (consensus); a randomised Byzantine agreement protocol (byzantine); the randomised mutual exclusion protocols of Pnueli \& Zuck (mutex) and Rabin (rabin); a simplified model of the Fibroblast Growth Factor signalling pathway $(f g f)$; a peer-to-peer protocol based on BitTorrent (peer2peer); and Dolev et al.'s minimum space shared memory leader election protocol (leader). All models, properties and references, as well as binary and source code versions of GRIP, can be found at [15].

We compare the performance of GRIP with PRISM and PRISM-symm [10], a symmetry reduction tool, implemented in PRISM, that constructs a symbolic (MTBDD) representation for the unreduced model and then performs symmetry reduction at the level of the MTBDD data structure. Experiments were performed on a $2.40 \mathrm{GHz}$ PC with 2 GB RAM, running PRISM version 3.2 under Linux.

\section{A. Results}

Experimental results are shown in Fig. 5. For each family of specifications, \#M denotes the number of symmetric modules in a particular specification and $|S(M)|$ the number of local states of each symmetric module (all of our examples consist of multiple instances of a single module type).

Columns 3-7 give the sizes of the unreduced and reduced models (produced by either GRIP or PRISM-symm) and the model storage requirements. The latter is given in terms of the number of nodes in the MTBDD used by PRISM to represent the model (one node uses 20 bytes of storage).

Columns 8-13 give the time required to build each model and perform probabilistic model checking. For the model building process, all three tools start from the same highlevel model specification. For PRISM, this specification is translated into an MTBDD representation of the corresponding probabilistic model, the set of all reachable states is computed, and all unreachable states are removed. PRISM-symm performs the same model construction process, and then applies symmetry reduction to the MTBDD representation. GRIP first applies the language-level symmetry reduction 
process described in this paper then passes the resulting generic model specification to PRISM for construction. The models constructed by each tool are model checked using PRISM, applying the fastest available technique of the tool.

\section{B. Discussion}

As is clearly illustrated by the table in Fig. 5, the reductions in state-space provided by symmetry reduction are substantial. This has a significant effect on the efficiency or feasibility of applying probabilistic model checking. This is because, in many cases, when numerical computations are performed on the model, storage proportional to the number of states is required (even when using compact, symbolic techniques to store the model itself). For some examples symmetry reduction permits verification where it was previously impossible due to insufficient memory or prohibitively long solution times.

Another important factor for the efficiency of model checking is the size of the MTBDD representing the model. In 4 out of 7 case studies, GRIP produces a smaller MTBDD than both PRISM and PRISM-symm. In other cases both GRIP and PRISM-symm produce a larger MTBDD than PRISM, despite the much smaller state-space. This is caused by the loss of regularity in the probabilistic model, once symmetric states have been collapsed.

From Fig. 5, we see that in terms of model construction time GRIP also performs better than the other tools on 4 out of 7 examples. In two cases GRIP performs poorly - this is due to the size of the MTBDDs produced for these models.

A comparison of GRIP and PRISM-symm in terms of verification time is also interesting. For three case studies, model checking is much faster on the GRIP-reduced models, despite the fact the symmetric quotient model is the same. For two of these (consensus and byzantine) the cause is a side-effect of the PRISM-symm reduction process whereby duplicated probability distributions can appear in the quotient MDP. This has no effect on the correctness of verification, but can slow down the process. On the third case study (rabin), the superior performance of GRIP is due to the fact that it results in a much smaller MTBDD.

On another example (peer2peer), model checking of the GRIP-reduced model is slower than for PRISM-symm. Here, the large number of variables in the GRIP model results in an MTBDD which is expensive to manipulate. Typically, GRIP is faster for systems comprising a large number of simple modules, whereas PRISM-symm will perform better on a small number of more complex modules.

\section{RELATED WORK}

Symmetry reduction techniques for model checking were originally proposed in [1], [2], [3] and have been studied extensively since (see [4] for a survey). The use of generic representatives in model checking was proposed in [5] and extended in [6], [16]. The approach was extended to probabilistic model checking [7] via the definition of Symmetric PRISM, a precursor to SPSL. A tool paper describing a preliminary version of the GRIP tool appeared as [8].

Another approach to symmetry reduction for probabilistic model checking is given in [10], which performs a modellevel reduction built into the symbolic implementation of the PRISM tool. This approach is based on dynamic symmetry reduction for non-probabilistic model checking [17], [18]. Language-level exploitation of symmetry has also been explored for several other probabilistic modelling formalisms, for example, stochastic process algebrae [19], stochastic Petri nets [20] and stochastic activity networks [21].

\section{CONCLUSions ANd Future Work}

We have introduced SPSL, a simple but general language for specifying symmetric, probabilistic systems. We have provided an algorithm, based on the generic representatives approach to symmetry reduction, which translates an SPSL specification into an equivalent generic form. This can then be used to model check symmetric properties of the original model, with considerable increase in efficiency thanks to the reduced size of the generic model. SPSL is a much richer language than those provided by existing generic representatives techniques, allowing for example multiple families of symmetric processes, multiple local variables per process and complex expressions over these variables. We have implemented our techniques in a tool, GRIP, which targets the PRISM modelling language, and have presented very positive results for a large set of case studies.

We plan to extend this work in a number of directions. Firstly, some useful features of the PRISM modelling language, notably synchronisation between modules, have not yet been incorporated into SPSL. We hope to extend our generic representatives approach to include these. Secondly, the fact that these techniques are based on language-level translations raises a number of interesting issues concerning, for example, the provision to the user of probabilistic counterexamples or optimal adversaries. Finally, we plan to investigate optimising symbolic implementations of probabilistic model checking for generic representatives, for example using zero-suppressed variants of binary decision diagrams.

\section{ACKNOWLEDGEMENT}

Alastair F. Donaldson, David Parker and Alice Miller are supported by EPSRC projects EP/G051100, EP/D07956X and EP/E032354 respectively.

\section{REFERENCES}

[1] E. Clarke, S. Jha, R. Enders, and T. Filkorn, "Exploiting symmetry in temporal logic model checking." Formal Methods in System Design, vol. 9, no. 1/2, pp. 77-104, 1996.

[2] E. Emerson and A. Sistla, "Symmetry and model checking." Formal Methods in System Design, vol. 9, no. 1/2, pp. 105131, 1996. 


\begin{tabular}{|c|c|c|c|c|c|c|c|c|c|c|c|c|}
\hline \multirow[b]{2}{*}{$\begin{array}{c}\text { Case study } \\
(|S(M)|)\end{array}$} & \multirow[b]{2}{*}{$\# M$} & \multicolumn{2}{|c|}{ Model size (states) } & \multicolumn{3}{|c|}{ Model storage (MTBDD nodes) } & \multicolumn{3}{|c|}{ Build time (sec.) } & \multicolumn{3}{|c|}{ Model check time (sec.) } \\
\hline & & $\begin{array}{l}\text { Full } \\
\text { model }\end{array}$ & $\begin{array}{l}\text { Symm. } \\
\text { reduced }\end{array}$ & PRISM & $\begin{array}{l}\text { PRISM } \\
\text {-symm }\end{array}$ & GRIP & PRISM & $\begin{array}{l}\text { PRISM } \\
\text {-symm }\end{array}$ & GRIP & PRISM & $\begin{array}{l}\text { PRISM } \\
\text {-symm }\end{array}$ & GRIP \\
\hline & 8 & $6.1+\mathrm{e} 7$ & 46,482 & 15,681 & 14,078 & 12,008 & 1.18 & 1.48 & 2.90 & 20,013 & 12.5 & 7.57 \\
\hline consensus & 12 & $1.2 \mathrm{e}+11$ & 339,729 & 50,431 & 45,352 & 27,766 & 5.55 & 6.34 & 5.89 & $>24 h$ & 183.0 & 99.8 \\
\hline \multirow[t]{2}{*}{ (6) } & 16 & $2.1 \mathrm{e}+14$ & $1.5 \mathrm{e}+6$ & 116,133 & 104,902 & 60,107 & 15.3 & 21.4 & 14.4 & $>24 h$ & 1,380 & 734.3 \\
\hline & 8 & $6.4 \mathrm{e}+8$ & 298,993 & 713,143 & 167,587 & 167,372 & 19.1 & 21.8 & 15.6 & 92.0 & 8.53 & 7.88 \\
\hline byzantine & 12 & $1.0 \mathrm{e}+13$ & $8.0 e+6$ & $4.3 e+6$ & 937,484 & 646,455 & 132.9 & 160.5 & 33.6 & mem-out & 91.1 & 57.3 \\
\hline \multirow[t]{2}{*}{ (10) } & 16 & $1.9 \mathrm{e}+16$ & $1.1 \mathrm{e}+8$ & $1.3 \mathrm{e}+7$ & $3.0 \mathrm{e}+6$ & $1.9 \mathrm{e}+6$ & 831.0 & 1,013 & 113.5 & mem-out & 429.6 & 190.5 \\
\hline & 4 & 201,828 & 11,130 & 65,624 & 96,559 & 53,171 & 1.19 & 2.52 & 14.6 & 0.11 & 0.18 & 0.11 \\
\hline rabin & 6 & $1.3 e+8$ & 356,592 & 206,213 & 408,291 & 185,943 & 6.22 & 14.3 & 42.2 & 0.46 & 0.60 & 0.45 \\
\hline \multirow[t]{2}{*}{$(17)$} & 8 & $4.5 \mathrm{e}+10$ & $4.1+\mathrm{e} 6$ & 381,184 & 796,324 & 430,901 & 17.5 & 41.2 & 87.3 & 0.91 & 1.74 & 1.13 \\
\hline & 5 & $4.6 \mathrm{e}+6$ & 63,756 & 224,843 & 440,445 & 601,627 & 10.5 & 18.2 & 17.2 & 1009.0 & 11.5 & 13.2 \\
\hline$f g f$ & 6 & $9.6 e+7$ & 283,360 & 522,063 & $1.0 \mathrm{e}+6$ & $1.2 \mathrm{e}+6$ & 39.9 & 64.4 & 39.6 & $>24 h$ & 69.2 & 72.6 \\
\hline \multirow{2}{*}{ (19) } & 7 & $2.0 \mathrm{e}+9$ & $1.1 \mathrm{e}+6$ & $1.1 \mathrm{e}+6$ & $2.2 \mathrm{e}+6$ & $2.3 e+6$ & 110.2 & 180.9 & 94.2 & mem-out & 342.1 & 354.2 \\
\hline & 5 & $3.4 \mathrm{e}+7$ & 376,992 & 26,266 & 101,630 & 157,476 & 0.10 & 1.06 & 3.93 & 1055.1 & 9.10 & 24.8 \\
\hline peer2peer & 6 & $1.1 \mathrm{e}+9$ & $2.3 e+6$ & 40,591 & 189,704 & 247,122 & 0.12 & 3.07 & 4.77 & mem-out & 72.8 & 174.8 \\
\hline \multirow[t]{2}{*}{ (32) } & 7 & $3.4 \mathrm{e}+10$ & $1.3 \mathrm{e}+7$ & 54,916 & 306,123 & 355,721 & 0.40 & 4.61 & 5.40 & mem-out & 472.4 & 1,049 \\
\hline & 60 & $4.2 \mathrm{e}+28$ & 1,891 & 44,780 & 32,989 & 1,602 & 3.10 & 30.4 & 1.94 & 72.2 & 3.83 & 0.07 \\
\hline & 100 & $5.2 \mathrm{e}+47$ & 5,151 & 122,660 & 90,989 & 2,858 & 11.7 & 259.1 & 2.04 & 997.0 & 20.0 & 0.19 \\
\hline $\begin{array}{c}\text { leader } \\
\text { (3) }\end{array}$ & 140 & $6.3 e+66$ & 10,011 & 238,940 & 177,789 & 4,618 & 32.5 & 1,081 & 2.24 & 6,241 & 60.5 & 0.42 \\
\hline \multirow{3}{*}{$\begin{array}{c}\text { mutex } \\
\text { (16) }\end{array}$} & 30 & $1.0 \mathrm{e}+31$ & $7.3 \mathrm{e}+8$ & 258,937 & 135,428 & 97,977 & 235.8 & 257.4 & 99.5 & 146.8 & 14.9 & 10.2 \\
\hline & 36 & $1.2 \mathrm{e}+37$ & $3.1 \mathrm{e}+9$ & 373,159 & 192,839 & 137,758 & 521.0 & 558.9 & 458.6 & 431.8 & 43.7 & 29.1 \\
\hline & 42 & $1.4 \mathrm{e}+43$ & $1.1 \mathrm{e}+10$ & 508,117 & 260,294 & 166,237 & 1,049 & 1,052 & 2,218 & 635.5 & 40.5 & 32.2 \\
\hline
\end{tabular}

Figure 5. Experimental results for GRIP, PRISM and PRISM-symm.

[3] C. Ip and D. Dill, "Better verification through symmetry." Formal Methods in System Design, vol. 9, no. 1/2, pp. 4175,1996

[4] A. Miller, A. Donaldson, and M. Calder, "Symmetry in temporal logic model checking," ACM Computing Surveys, vol. 38 , no. 3 , p. 8,2006 .

[5] E. Emerson and R. Trefler, "From asymmetry to full symmetry: New techniques for symmetry reduction in model checking." in Proc. CHARME'99, ser. LNCS, vol. 1703. Springer, 1999, pp. 142-156.

[6] E. Emerson and T. Wahl, "On combining symmetry reduction and symbolic representation for efficient model checking." in Proc. CHARME'03, ser. LNCS, vol. 2860. Springer, 2003, pp. 216-230.

[7] A. Donaldson and A. Miller, "Symmetry reduction for probabilistic model checking using generic representatives." in Proc. ATVA'06, ser. LNCS, vol. 4218. Springer, 2006, pp. 9-23.

[8] A. Donaldson, A. Miller, and D. Parker, "GRIP: Generic representatives in PRISM," in Proc. QEST '07. IEEE Computer Society, 2007, pp. 115-116.

[9] A. Hinton, M. Kwiatkowska, G. Norman, and D. Parker, "PRISM: A tool for automatic verification of probabilistic systems." in Proc. TACAS'06, ser. LNCS, vol. 3920. Springer, 2006, pp. 441-444.

[10] M. Kwiatkowska, G. Norman, and D. Parker, "Symmetry reduction for probabilistic model checking." in Proc. CAV'06, ser. LNCS, vol. 4144. Springer, 2006, pp. 234-248.

[11] H. Hansson and B. Jonsson, "A logic for reasoning about time and reliability," Formal Aspects of Computing, vol. 6, no. 5, pp. 512-535, 1994
[12] A. Bianco and L. de Alfaro, "Model checking of probabilistic and nondeterministic systems," in Proc. FSTTCS'95, ser. LNCS, vol. 1026. Springer, 1995, pp. 499-513.

[13] A. Miller and A. Donaldson, "Property preservation in quotient structures." Department of Computing Science, University of Glasgow, Technical Report TR-2008-270, 2008.

[14] A. Donaldson, A. Miller, and D. Parker, Language-level Symmetry Reduction for Probabilistic Model Checking, extended version with proofs, 2009

www.allydonaldson.co.uk/papers/2009/QEST_full.pdf.

[15] GRIP Website, University of Glasgow/PRISM group, 2008, www.prismmodelchecker.org/grip/.

[16] E. Emerson and T. Wahl, "Efficient reduction techniques for systems with many components." Electr. Notes Theor. Comput. Sci., vol. 130, pp. 379-399, 2005.

[17] E. Emerson and T. Wahl, "Dynamic symmetry reduction." in Proc. TACAS'05, ser. LNCS, vol. 3440. Springer, 2005, pp. 382-396.

[18] T. Wahl, N. Blanc, and E. Emerson, "SVISS: Symbolic verification of symmetric systems," in Proc. TACAS'08, ser LNCS, vol. 4963. Springer, 2008, pp. 459-462.

[19] S. Gilmore, J. Hillston, and M. Ribaudo, "An efficient algorithm for aggregating PEPA models," IEEE Trans. Software Eng., vol. 27, no. 5, pp. 449-464, 2001.

[20] G. Chiola, C. Dutheillet, G. Franceschinis, and S. Haddad, "Stochastic well-formed coloured nets for symmetric modeling applications," IEEE Trans. Computers, vol. 42, no. 11, 1993.

[21] J. Meyer and W. Sanders, "Reduced base model construction methods for stochastic activity networks," IEEE J. Selected Areas in Comm., vol. 9, no. 1, pp. 25-36, 1991. 